\title{
Temperature Dependence of the Electrical Resistivity and Thermoelectric Power of Rare Earth Substituted $\mathrm{Cu}-\mathrm{Cd}$ ferrite
}

\author{
A.A. Sattar \\ Physics Department, Faculty of Science, Ain Shams University, \\ 11566 Abbassia, Cairo-Egypt. \\ E-mail:Adel_Sattar@hotmail.com
}

\begin{abstract}
Copper-cadmium ferrite substituted with different rare earth ions $R(R=$ $L a, S m, G d$ and $D y)$ are investigated. The temperature dependence of the electrical resistivity $(\rho)$ and thermoelectric power $(\alpha)$ are measured and hence the mobility is calculated. The electrical resistivity is increased with increasing the ionic radius of samples substituted with $R=D y, G d$ and Sm while it decreased in case La substituted sample. The thermoelectric power is found to be temperature independent. The behavior of $\rho, \alpha$ and the values of the activation energy of the resistivity $E_{\rho}$ support the small polaron hopping conduction mechanism.
\end{abstract}

\section{Introduction:}

High resistivity ferrites with low eddy current losses are required from the technological applications point of view. The electrical resistivity of ferrites has been normally found to increase on doping or substituting with other oxides. Thus the electrical resistivity of copper ferrite was increased by substituting divalent ions $\left(\mathrm{Ni}^{2+}\right.$ or $\left.\mathrm{Cd}^{2+}\right)$ [1], trivalent ions $\left(\mathrm{Cr}^{3+}\right)$ [2] and tetravalent ions $\left(\mathrm{Ti}^{4+}\right)$ [3]. In the last few years many workers investigated the magnetic and electrical properties of ferrites substituted by rare earth oxides [49]. Kolekar et al [10] studied the electrical properties of $\mathrm{Gd}$ substituted $\mathrm{Cu}-\mathrm{Cd}$ mixed ferrite and they found that the dc electrical resistivity is increased. In previous work [11], the electrical properties of $\mathrm{Cu}-\mathrm{Zn}$ ferrite substituted with different rare earth oxides were investigated and the electrical resistivity was also increased for all substituted samples. It is known that $\mathrm{Zn}^{2+}$ and $\mathrm{Cd}^{2+}$ ions have similar effects, both are diamagnetic and prefer to occupy the tetrahedral sites only [12]. Therefore, it was of interest in the present article to investigate the electrical properties of $\mathrm{Cu}-\mathrm{Cd}$ ferrite substituted with different rare earth oxides. The electrical resistivity and thermoelectric power are measured. The latter allows better understanding of the conduction mechanism. 


\section{2 . Experimental Techniques:}

Samples with the chemical formula $\mathrm{Cu}_{0.7} \mathrm{Cd}_{0.3} \mathrm{Fe}_{2-x} \mathrm{R}_{\mathrm{x}} \mathrm{O}_{4}(\mathrm{R}=\mathrm{La}, \mathrm{Sm}$, Gd and Dy ; $x=0.0$ and 0.02) were prepared by the standard ceramic method. High purity oxides, $99.9 \%$, of $\mathrm{CuO}, \mathrm{CdO}, \mathrm{Fe}_{2} \mathrm{O}_{3}$ and $\mathrm{R}_{2} \mathrm{O}_{3}$ were mixed according to their molecular weights to produce the required composition. All samples were presintered at $900{ }^{\circ} \mathrm{C}$ for $6 \mathrm{~h}$ and then milled again to fine powders. The powders were then pressed into pellets and toroids under a pressure of $3 \times 10^{8} \mathrm{~Pa}$. All samples were finally sintered at $1100{ }^{\circ} \mathrm{C}$ for $2 \mathrm{~h}$ and slowly cooled in the furnace to room temperature. X-ray diffraction patterns were recorded on fine powders using a diffractometer of type X'pert - MPD with $\mathrm{Cu} \mathrm{K} \mathrm{K}_{\alpha}$ radiation. The toroidal samples were used to measure the initial permeability, as described in details in previous work [9], from which the Curie temperature was determined. Using disc samples of average thickness 2-3 mm, the dc electrical resistivity was measured as a function of temperature by the two probe method. In- $\mathrm{Hg}$ was used as a contact material. A small constant current $(<10 \mathrm{~mA})$ was passed through the sample to avoid the Joule heating. For thermoelectric power measurement, each sample was fitted directly against $\mathrm{Pt}$ electrodes and a temperature gradient of $12{ }^{\circ} \mathrm{C} / \mathrm{cm}$ was kept constant across the sample. The temperatures of both surfaces of the pellet were measured with the aid of Chromel-Alumel thermocouples. The sign of the thermoelectric voltage $\Delta \mathrm{V}$ was taken as that of the cold surface. The thermoelectric power $\alpha$ is calculated as $\alpha=\Delta \mathrm{V} / \Delta \mathrm{T}$ where $\Delta \mathrm{T}$ is the temperature difference between the two surfaces of the sample. The error in the measurements of the electrical resistivity and thermoelectric power is $2 \%$ and $3 \%$ respectively.

\section{Results and discussion:}

X-rays diffraction patterns revealed spinels of single phase. Fig. (1) shows the variations of the electrical resistivity $\rho$ (represented as $\log \rho$ ) against temperature (represented as $10^{3} / \mathrm{T}$ ). It is clear that the electrical resistivity of all investigated samples decreases with increasing temperature, i.e exhibits semiconducting behavior, according to the well known relation

$$
\rho=\rho_{\mathrm{o}} \exp (\mathrm{E} \rho / \mathrm{kT})
$$

where $\rho_{\mathrm{o}}$ is a constant and $\mathrm{E} \rho$ is the activation energy of the resistivity. From Fig. 1, one may also notice that there are two regions of conduction with different activation energies. The change from one region to the other is not sharp but it is a gradual one. The activation energy in the high temperature range is larger than that in the lower temperature range. The increase in E $\rho$ is observed at certain temperatures depending on the composition. The values of these temperatures at which $\mathrm{E} \rho$ are increased, $\mathrm{T} \rho$, are determined from the 
intersection of the linear parts drawn for each conduction region and are given in Table 1. Fig. (2) illustrates the changes of the initial permeability $\mu_{\mathrm{i}}$ with temperature. The Curie temperature $T_{c}$ is determined from the intersection of $\mu_{i}$ curve with the temperature axis. The values of $T_{c}$ are also given in Table 1 . Comparing the values of $T \rho$ and $T_{c}$, it is clear that there is an agreement between the two temperatures. Therefore, the increase in $E \rho$ at $T=T_{c}$ can be attributed to a change of magnetic ordering i.e a change from a ferrimagnetic to a paramagnetic state. The values of E $\rho$ are determined from the slope of the straight line of each curve using the least square fitting. The calculated values of $E \rho$ of ferrimagnetic phase $E_{f}$ and that in the paramagnetic phase $E_{p}$ are given in table 1. The effect of magnetic transition on the electrical properties was observed by many workers [13-16].It is known from thermodynamics that the magnetic transition, which is a second order transition, is accompanied by a change in the volume expansivity [17]. Such expansion increases the distances between the ions that are responsible for the conduction process and hence increases the activation energy of the resistivity in the paramagnetic state $E_{p}$ relative to that in the ferrimagnetic state $E_{f}$. From Table 1, it is valuable to notice that samples substituted with $\mathrm{R}=\mathrm{Dy}, \mathrm{Gd}$ and $\mathrm{Sm}$ have almost the same Curie temperatures $T_{c}$ and the un-substituted sample $(R=0)$ has the highest $T_{c}$ value. It is due to the fact that the substituted samples have the same $\mathrm{Fe}$ concentration which is lower than that contained in sample with $\mathrm{R}=0$ i.e the number of interactions will be smaller and meanwhile $\mathrm{R}-\mathrm{R}$ and $\mathrm{R}-\mathrm{Fe}$ interactions are weak.

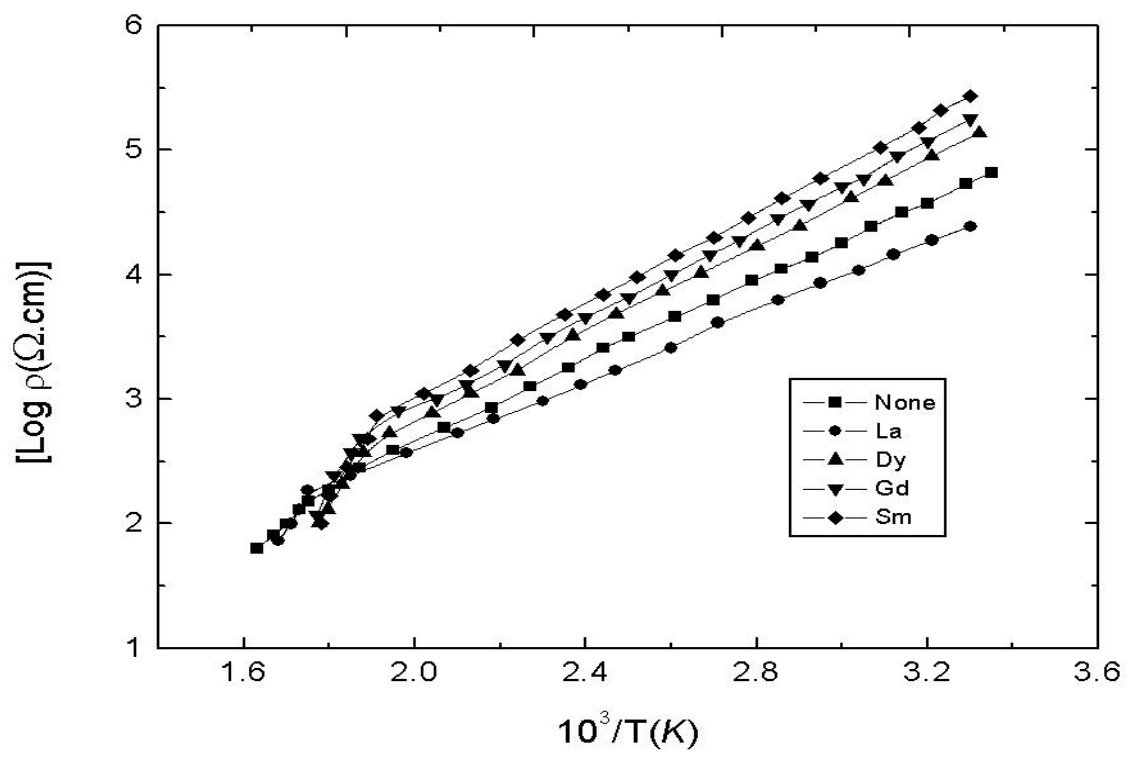

Fig.(1): Temperature dependence of the resistivity for different rare earth substituted cu-cd Ferrite. 


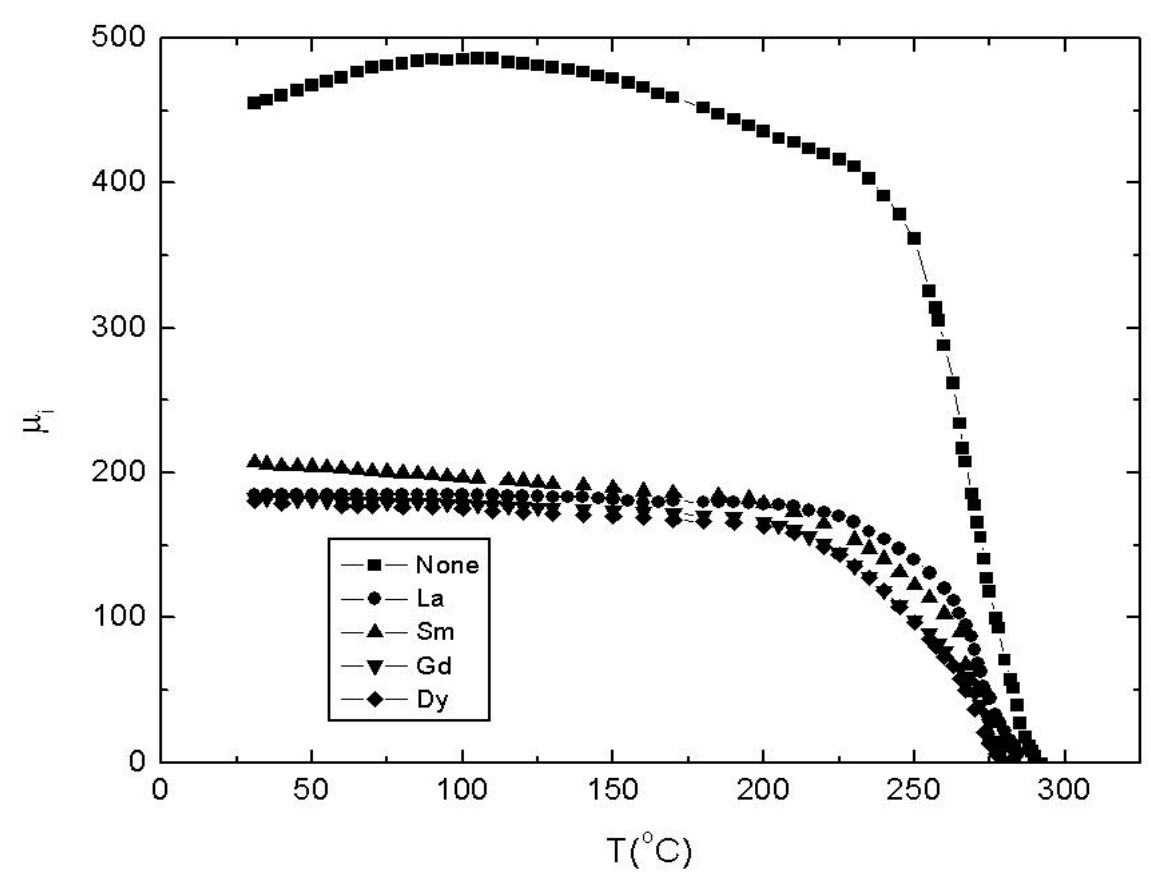

Fig.(2): Variations of the initial permeability $\mu_{i}$ with temperature for different rare earth substituted cu-cd Ferrite.

Table(1): Curie temperatures determined from resistivity, $\mathrm{T} \rho$, and from initial permeability, $T_{c}$, activation energies $E_{f}, E_{p}$ and $E_{\mu}$ for different substituted rare earth ion

\begin{tabular}{|c|c|c|c|c|c|}
\hline \multirow{2}{*}{$\mathrm{R}$ ion } & \multicolumn{2}{|c|}{ Curie temperature $(\mathrm{K})$} & \multicolumn{2}{|c|}{$\mathrm{E} \rho(\mathrm{eV})$} & \multirow{2}{*}{$\mathrm{E}_{\mu}(\mathrm{eV})$} \\
\cline { 2 - 5 } & $\mathrm{T}_{\rho}$ & $\mathrm{T}_{\mathrm{c}}$ & $\mathrm{E}_{\mathrm{f}}$ & $\mathrm{E}_{\mathrm{p}}$ & 0.308 \\
\hline None & 571 & 560 & 0.314 & 0.63 & 0.346 \\
\hline $\mathrm{Dy}$ & 535 & 553 & 0.346 & 0.83 & 0.346 \\
\hline $\mathrm{Gd}$ & 532 & 551 & 0.35 & 0.92 & 0.35 \\
\hline $\mathrm{Sm}$ & 524 & 552 & 0.365 & 0.85 & 0.365 \\
\hline $\mathrm{La}$ & 570 & 555 & 0.28 & 0.78 & 0.279 \\
\hline
\end{tabular}

The variations of the electrical resistivity, at room temperature, and the activation energy in the ferrimagnetic region, $\mathrm{E}_{\mathrm{f}}$, with ionic radius of the substituted ion are given in Fig. (3). The values of the ionic radii are taken from reference [18]. It is seen that, relative to un-substituted sample, both $\log \rho$ and $E_{f}$ increase with increasing the ionic radius, except La substituted sample. This behavior could be explained assuming that the La ions reside at the grain boundaries while the other substituted rare earth ions enter into the B-sites of 
the spinel structure. Such assumption is proved experimentally through the results reported in [6,7]. The presence of rare earth ion at the B-site will increase the separation between $\mathrm{Fe}^{2+}$ and $\mathrm{Fe}^{3+}$ in proportion to its ionic radius. Thus it impedes the electron transfer between $\mathrm{Fe}^{2+}$ and $\mathrm{Fe}^{3+}$ i.e it increases the activation energy and hence the electrical resistivity according to its ionic radius. The presence of $\mathrm{La}$ ion at the grain boundaries seems to facilitate the electron transfer between $\mathrm{Fe}^{2+}$ and $\mathrm{Fe}^{3+}$ in the B-sites.

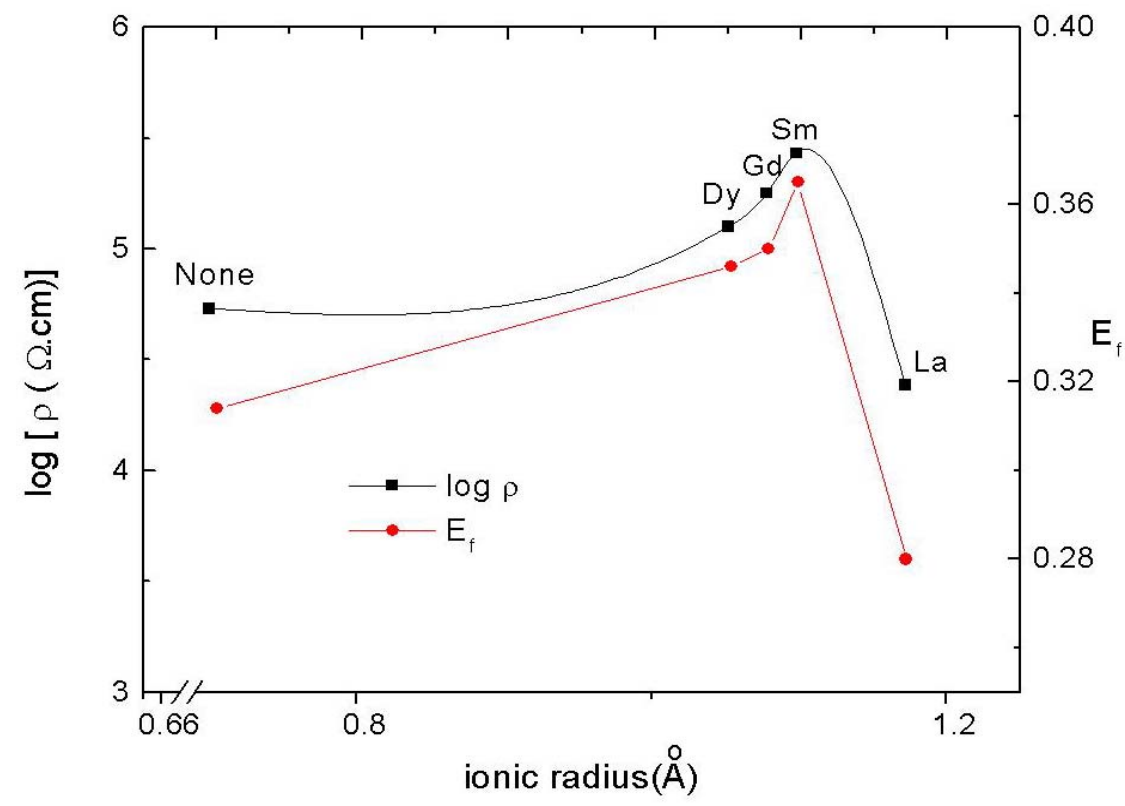

Fig.(3): Changes of the electrical resistivity $\rho$ and activation energy in the ferrimagnetic region $\mathrm{E}_{\mathrm{f}}$ with the ionic radii of the substituted ions.

Figure (4) illustrates the changes of thermoelectric power $(\alpha)$ with temperature. It is obvious that the thermoelectric power is almost independent of temperature for all investigated samples with slight changes in $(\alpha)$ near the Curie temperatures. It is clear also that the thermoelectric power is negative, in the whole studied temperature range, which indicates that the majority of charge carriers are electrons. Similar results were obtained for rare earth substituted $\mathrm{Cu}$ $-\mathrm{Zn}$ ferrites [11]. Moreover, it is seen that the unsubstituted sample has the highest absolute value of $(\alpha)$ while the Dy, Gd and Sm substituted samples have almost the same values of $(\alpha)$. The La substituted sample has the lowest value. These results could be explained according to the relation [19].

$$
\alpha=-2.303 \mathrm{~K} / \mathrm{e} \log \left(\mathrm{Fe}^{3+} / \mathrm{Fe}^{2+}\right)=-2.303 \mathrm{~K} / \mathrm{e} \log \left(\mathrm{N}_{\mathrm{o}} / \mathrm{n}\right)
$$




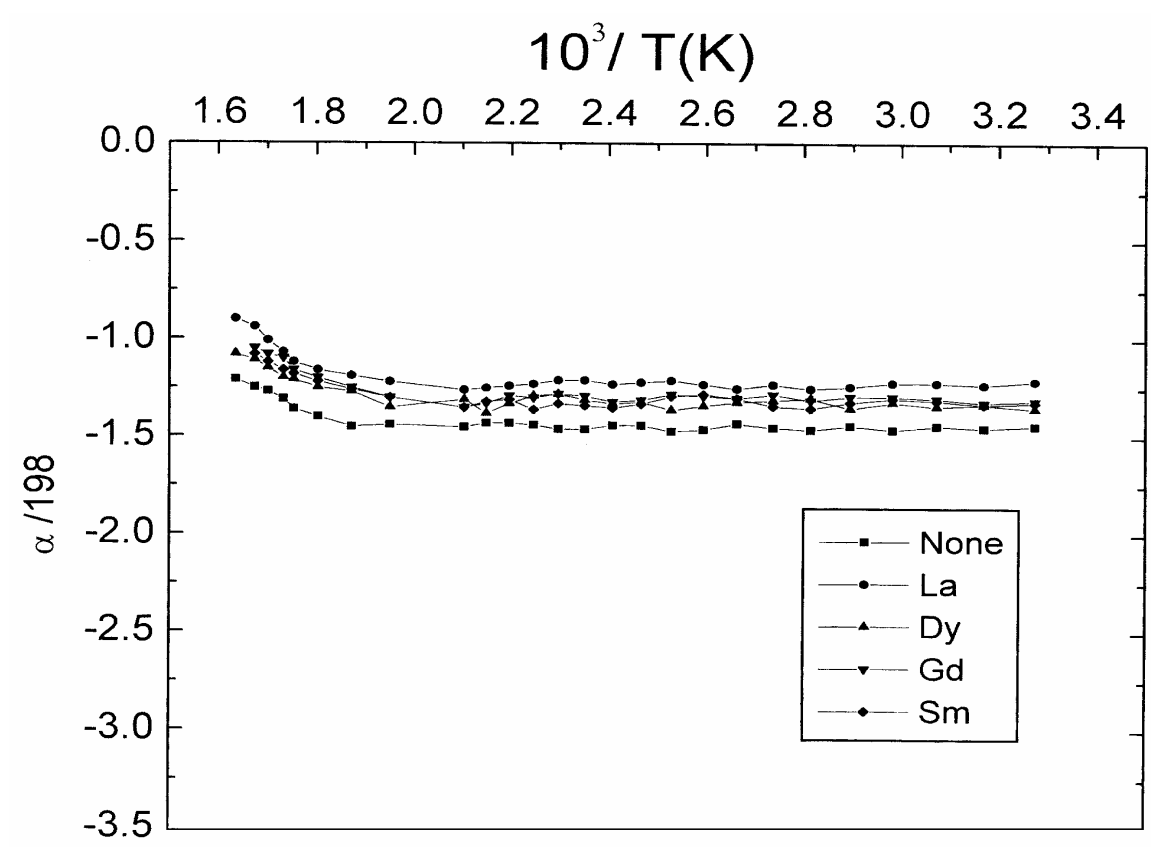

Fig.(4): Temperature dependence of the thermoelectric power $\alpha$ for different rare earth substituted cu-cd Ferrite.

where $\mathrm{N}_{\mathrm{o}}$ and $\mathrm{n}$ are the concentrations of $\mathrm{Fe}^{3+}$ and $\mathrm{Fe}^{2+}$ ions in octahedral sites respectively. The value of $2.303 \mathrm{~K} / \mathrm{e}$ is equal to $198 \mu \mathrm{V} / \mathrm{K}$. The $\mathrm{Fe}^{2+}$ ions result from a partial reduction of $\mathrm{Fe}^{3+}$ to $\mathrm{Fe}^{2+}$ at elevated temperature and due to $\mathrm{Cd}$ volatilization during the sintering process [20]. Thus, the presence of $\mathrm{Fe}^{2+}$ ions accounts on the negative sign of thermoelectric power. It is expected that the $\mathrm{Fe}^{2+}$ concentration will be the same for all substituted samples as the samples are heat treated simultaneously. Moreover, in our chemical formula, $0.02 \mathrm{Fe}^{3+}$ ions are replaced by $0.02 \mathrm{R}$ ions. Therefore, it is expected that $\mathrm{Fe}^{3+} / \mathrm{Fe}^{2+}$ ratio, for substituted samples, will be constant. Thus according to eq. (2), these samples must have the same values of $(\alpha)$ as we are found experimentally for samples with $\mathrm{R}=\mathrm{Dy}, \mathrm{Gd}$ and $\mathrm{Sm}$. For the unsubstituted sample, $\mathrm{Cu}_{0.7} \mathrm{Cd}_{0.3} \mathrm{Fe}_{2} \mathrm{O}_{4}$, the $\mathrm{Fe}^{3+}$ ion concentration is higher than that of the substituted samples and according to eq. (2), it must has the highest absolute value of $(\alpha)$ as shown in figure 4. Similar results were reported for $\mathrm{Nd}^{3+}$ substituted $\mathrm{Zn}-\mathrm{Mg}$ ferrite [6]. For the La substituted sample, in addition to the decrease of $\mathrm{Fe}^{3+}$ ion concentration, the presence of $\mathrm{La}$ ions at the grain boundaries enhances the electron transfer between $\mathrm{Fe}^{2+}$ and $\mathrm{Fe}^{3+}$ which accounts on the lowest absolute values of $(\alpha)$ for this sample. 
The crystal structure of ferrites shows that the cations, either in tetrahedral or in octahedral sites, are surrounded by oxygen anions and to a first approximation can be treated as isolated from each other. Thus the localized electron model is more appropriate to discuss the conduction mechanism in ferrite rather than the band model. Such a mechanism is distinguishable from the band conduction mechanism by

(1) A temperature independent thermoelectric power

(2) A thermally activated mobility

Our results concerning the thermoelectric power supports the localized model (hopping mechanism) where the thermoelectric power is found to be independent of temperature. Using eq. (2) for $\alpha$ and the electrical resistivity $\rho$ ( $\rho$ $=1 /$ ne $\mu$ ) an expression for the mobility is derived as

$$
\mu=\exp (\alpha / 198) / \mathrm{eN} \mathrm{N}_{\mathrm{o}}
$$

where $\mathrm{N}_{\mathrm{o}}=1.3 \times 10^{22}$ atoms $/ \mathrm{cm}^{3}$. The values of $\alpha$ and $\rho$ at the same temperature are substituted in eq.(3) and the mobility is calculated. The temperature dependence of the mobility is shown in Fig. (5) and it follows the relation

$$
\mu=\mu_{\mathrm{o}} \exp \left(-\mathrm{E}_{\mu} / \mathrm{KT}\right)
$$

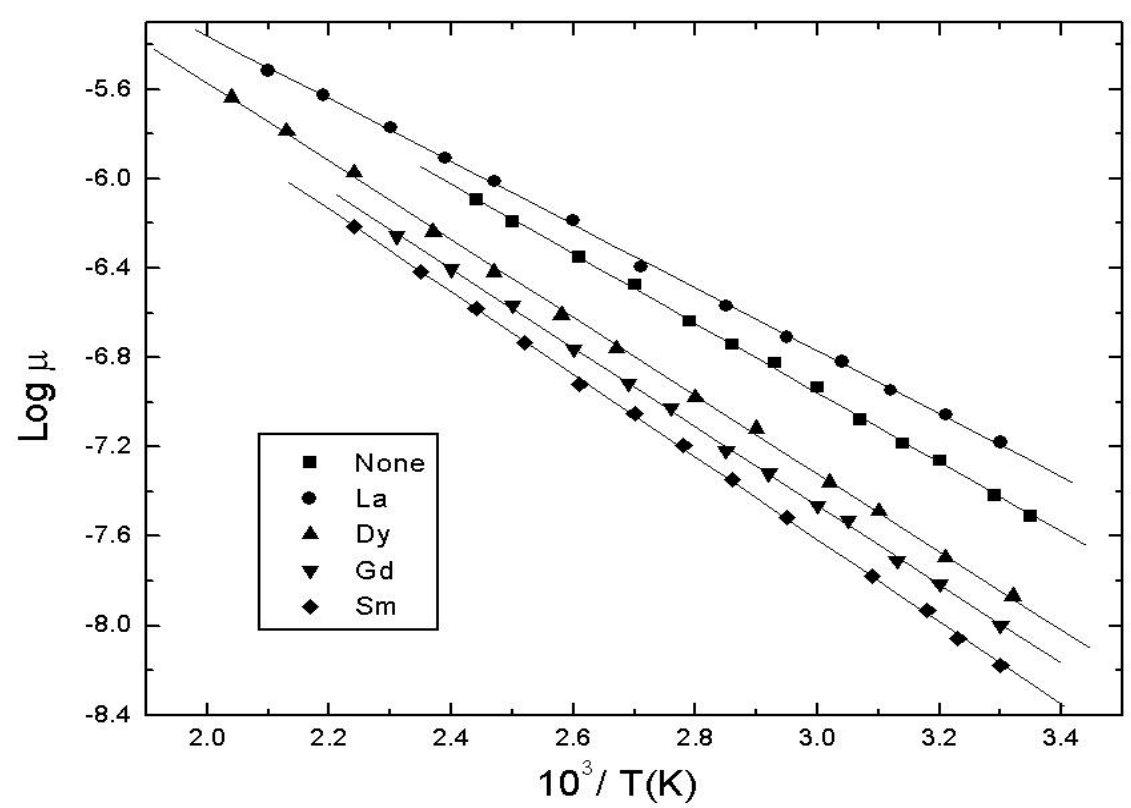

Fig.(5): Variations of the mobility $\mu$ with temperature for different rare earth substituted cu-cd Ferrite. 
where $\mu_{o}$ is a constant and $E_{\mu}$ is the activation energy of the mobility. The values of $E_{\mu}$ are calculated using the least square method and are given in table 1. Comparing the values of $E_{\rho}$ and $E_{\mu}$ one finds that both energies are identical. This means that the energy is consumed mainly in moving the electrons and not for generation of further charge carriers. Moreover, the activation energies given in table 1 are greater than $0.28 \mathrm{eV}$ while the transition energy between $\mathrm{Fe}^{2+}$ and $\mathrm{Fe}^{3+}$ is $0.2 \mathrm{eV}$ [19]. According to the work reported in [19], it was suggested that if $E_{\rho}$ is higher than $0.2 \mathrm{eV}$ then the conduction mechanism is predominantly due to small polaron than the electron hopping mechanism. Thus these results support the small polaron hopping mechanism.

\section{Conclusion:}

1- The electrical resistivity is increased due to increasing the separation between the magnetic ions while the number of charge carriers remained constant.

2- The constancy of thermoelectric power and the increase of the mobility with temperature support the hopping conduction mechanism.

\section{References:}

1. A.A.Ghani Awad, Phys. State Sol. (a), 81, K155 (1984)

2. M.M.Mosaad, M.A.Ahmed, M.A.El-Hiti and S.M.Attia, J.Mag. Mag. Mat. 150, 51(1995)

3. B.L.Patial, S.R. Sawant and S.A. Patial, phys. state. sol. (a), 133, 147(1992)

4. N. Rezlescu, E.Rezlescu, C Pasnicu and M.L.Craus, J. Phys. Condens. Matter, 6, 5707(1994)

5. J.L. Bhosale, S.N. Kulkarni, R. B. Sasmile and B.K. Chougule, Indian J. Pure and Appl. Phys., 33, 412(1995)

6. B. P. Ladgaonkar, P.N. Vasambekar and A.S.Vaingankar, Bull. Mater. Sci., 23 (2), 87(2000)

7. A.A.Sattar, A.H.Wafik and H.M.El-Sayed, Journal of Materials Science, 36, 4703 (2001)

8. A.A.Sattar, A.H.Wafik and H.M.El-Sayed, Phys. State Sol. (a) 186 (3)415 (2001)

9. A.A.Sattar, A.M.Samy, R.S.El-Ezza and A.E.Eatah, phys. stat. sol. (a), 193 No.1, 86(2002

10. C.b.Kolekar, P.N.Kamble, A.S.Vaingankar, J. Mag. Mag. Mat., 138, 211(1994)

11. A.A.Sattar and K.M.El-Shokrofy, Proc. $7^{\text {th }}$ Int. Conf. On Ferrites, ICF7, Bordeaux, France, J. Phys. IV 7, C1-245 (1997).

12. J. Smit and H.P.J. Wijn, Ferrites,Cleaver - Hume Press Ltd, p. 142 (1959) 
13. A.A.Sattar, J. Mat. Sci. Lett. 15, 1090(1996)

14. Josyulu and J.Sabhanadri, phys. state. sol. (a), 59, 323 (1980).

15. M.A.Ahmed, M.K. El-Nimer, A.Tawfik and A.M.El-Hasab, phys. stat. sol. (a), 123, 501 (1991)

16. M.G.Patil, V.C. Mahajan, B.V.Bhise, S.M.Chendke and S.A. Patil, phys. stat. sol. (a), 144, 415(1994)

17. M.W. Zemansky, Heat and Thermodynamics, Mc Graw - Hill, p. 460 (1968)

18. R.D. Shannon, Acta cryst., A32, 751(1976)

19. A.J.Bosmsn and H.J.Van Daal, Advances in Physics, 19 (77),1(1970)

20. Globus, H.Pascard and V.Cagan, J.de Physique, 38,C1-163 (1977) 